\title{
Impact of Signal and Image Processing, Communications and Networking
}

\section{Sukumar Senthilkumar*}

School of Computing Science and Engineering, Vellore Institute of Technology-University, Tamilnadu, India

Intensive research is still underway exclusively in the field of signal, image, and video processing which in turn explores engineering issues associated to the modeling of signals starting from the physics of the problem, developing and evaluating algorithms for extracting the necessary information from the signal, and the implementation of these algorithms on electronic and opto-electronic systems. In particular modern research areas include filter design, fast transforms, adaptive filters, spectrum estimation and modeling, sensor array processing, image processing, motion estimation from images, and the implementation of signal processing algorithms through appropriate technologies with real time applications in sonar, radar, speech, geophysics, computer-aided tomography, image restoration, robotic vision, pattern recognition, MPEG video compression for transmission and storage, print quality enhancement, and feature extraction etc. The use of image processing techniques and its associated algorithms in remote sensing is a remarkable area of research expertise including speech recognition and synthesis. The idea of video and image processing provides the ability to display and process high-resolution imagery with the aid of multiple systems that digitize, display, and process digital video. In order to carry out the specific task successfully it is necessary to have a complete suite of video distribution and editing equipment, a real-time MPEG encoder, and an ATM test bed network. Also, digital signal processing must be supported with speech processing, nonlinear DSP, neural networks, design of specialized signals, signal representation, and DSP architectures. In case of electronic imaging systems, image capturing, image rendering, and document processing can be performed with the help of high-resolution and large format printers, high-precision scanners, and high-performance workstations. Video and image compression, computer networks, multimedia authoring, media capture, wireless systems, and a wide variety of applications can also be achieved with the aid of multimedia test bed in addition to investigate and evaluate networked multimedia systems. The occurrence of several data processing technologies and high-end digital computers has enabled the ability to mathematically manipulate data which has formerly relied on the brain for processing such as speech, audio, images, and video. In particular, three major thrusts areas are signal processing which includes audio compression, speech recognition, statistical signal processing, speech synthesis, language identification, wireless sensor networks. Secondly, image processing which includes image enhancement, image compression, image reconstruction, image segmentation, image recognition, image transmission, image storage, remote sensing data analysis, medical image analysis and finally video processing which includes content-based video analysis, object tracking / recognition, visual surveillance, human activity recognition, and multimedia applications, etc. Data processing touches and solves a wide variety of engineering problems, from communication to humancomputer interface, to medical imaging and multimedia. It is well known that digital image processing is a rapidly evolving area with growing practical applications in both professional markets (computer-aided tomography is extensively employed in life sciences) and consumer markets (digital photography). Indeed research applications span a wide range of fields which includes medical imaging, collaborative / array / distributed signal processing, speech processing for recognition and synthesis, immersive audio, multimedia signal processing and compression, optical information processing, signal processing for communications, and other multimedia related technologies such as content-based representation and retrieval.

On the other hand, innovative research is still being carried out in computer communication networks area to offer novel techniques for design, analysis, and optimization of increasingly complex and demanding communication. In order to enhance the quality, novel switch architectures are being proposed by various researchers for highspeed packet-switched networks that integrate video, voice, and data etc. Mechanisms for scheduling, bandwidth allocation, error recovery, data compression, and access control for local and wide-area computer networks will provide improved performance and quality of service. Researchers are providing significant outputs in smart antennas, accurate GPS, improved modems, and efficient radar applications through the amalgamation of signal processing and communications expertise. For instance, a narrow-band modem may be deployed in intelligent transportation systems which in turn may provide high-performance wireless communication. In digital communication, spread spectrum technique intentionally expands the bandwidth of a signal transmission. Real time applications of spread spectrum technology are carried out to personal communications systems, multimedia networks, and digital battlefields which in turn enabled a shift in coding and modulation techniques. Though the communications, networking, signal \& image processing fields independent with each other but sometimes they are mutually exclusive. In other words research can be merged through wireless mobile and PCS communication, smart antennas, GPS, radar, speech recognition and synthesis, image processing and pattern recognition, print image quality, remote sensing, local and wide-area computer networks, and multimedia communication and processing. Finally, outputs from this field really influence the communication with cellular phones, faxes, and the internet etc. For instance, one can travel using GPS and intelligent highways; and the video, audio, and data that end user receive and transmit for personal entertainment and electronic commerce. It is mandatory to pin point out that new algorithms, architectures and applications have to be developed based on fundamental theory and applications to real-world data processing problems in order to overcome all hazards in all the diversified fields, provided a platform for the development of tools, techniques and systems to be used for the acquisition, analysis and extraction of information.

*Corresponding author: Sukumar Senthilkumar, School of Computing Science and Engineering, Vellore Institute of Technology-University, Vellore-632014 Tamilnadu, India, Tel: 91-416 2202811; E-mail: senthilkumar@vit.ac.in, ssenthilkumar1974@yahoo.co.in

Received March 09, 2014; Accepted March 12, 2014; Published March 14, 2014

Citation: Senthilkumar S (2014) Impact of Signal and Image Processing, Communications and Networking. J Electr Electron Syst 3: e114. doi:10.4172/23320796.1000e114

Copyright: ๑ 2014 Senthilkumar S. This is an open-access article distributed under the terms of the Creative Commons Attribution License, which permits unrestricted use, distribution, and reproduction in any medium, provided the original author and source are credited. 